\title{
Hope, Ethnic Pride, and Academic Achievement: Positive Psychology and Latino Youth
}

\author{
Derik Yager-Elorriaga', Kathy Berenson'2, Paula McWhirter ${ }^{1}$ \\ ${ }^{1}$ The University of Oklahoma, Norman, USA \\ ${ }^{2}$ Gettysburg College, Gettysburg, USA \\ Email: paulamcwhirter@ou.edu
}

Received 17 June 2014; revised 13 July 2014; accepted 6 August 2014

Copyright (C) 2014 by authors and Scientific Research Publishing Inc.

This work is licensed under the Creative Commons Attribution International License (CC BY). http://creativecommons.org/licenses/by/4.0/

C) (i) Open Access

\begin{abstract}
Previous studies have found that hope has beneficial effects in athletics, academics, physical health, and mental well being in majority populations. Given the challenges Latino youth face in the United States, ethnic identity and hope may be a powerful buffer from these negative stressors. The current study aimed to identify whether chronic levels of hope related to academic performance, whether an ethnic pride manipulation altered state hope levels, and whether there was a link between ethnic identity and chronic hope among a sample of Latino youth. Results indicated that GPA and chronic hope levels were not related, a manipulation to boost ethic pride increased state hope, and that ethnic identity was related to chronic levels of hope. The findings suggest that ethnic identity is an important contributor to hope levels.
\end{abstract}

\section{Keywords}

Hope, Latino Youth, Academic Performance, Ethnic Identity

\section{Hope, Ethnic Pride, and Academic Achievement in Latino Youth}

The 2010 census estimates that 50.5 million people of Latino origin reside in the United States, making Latinos the largest ethnic minority group in the nation. However, there have been a relatively limited number of psychological studies regarding Latinos. As Latino populations continue to grow, the lack of research on this group becomes of particular concern, especially considering the unique challenges ethnic minorities face in this country.

Latino's living in the United States experience various life stressors that contribute to the risk of developing psychological problems (McClure \& Pine, 2006). For example, for Latinos ages 16 and older, 32\% said that they, 
a family member, or close friend had experienced discrimination within the last 5 years based on their racial or ethnic background (Lopez, Morin, \& Taylor, 2009). Furthermore, when asked about specific instances of discrimination, $64 \%$ of Latino adults identified discrimination against Latinos in schools as a major problem, and 58\% said the same about the workplace (Lopez, Morin, \& Taylor, 2009).

Given the challenges Latino's face in this country, it is of extreme importance to find and expand areas of research focusing on promoting positive effects. Recently, a line of extremely promising research has focused on the concept of hope. For centuries, language has captured humanity's positive views of hope through various cultural sayings (e.g. "hope is the last thing lost"). Furthermore, inspirational quotes have exemplified how we use hope as motivational fuel, especially for our youth (e.g. "when all else fades, hope remains"). As abstract as hope has been, over the past decades psychologists have begun to operationalize hope and quantify its positive effects.

Snyder's (1994) hope model has become one of the most well-known conceptualizations of hope. According to Snyder's theory, people are goal-oriented, and have two distinct ways of thinking about how to achieve their goals (Snyder, 2002). The first mode of thinking, pathway thinking, refers to the perceived confidence in one's ability to generate viable routes (both primary and alternate), and overcome obstacles, in order to achieve one's goals. The second mode of thinking, agency thinking, refers to the perceived capacity to use one's pathways to reach desired goals, and is the motivational aspect in hope theory. Although pathway and agency thinking are distinct and each alone cannot sufficiently define hope, they are positively related and often interact (Chang \& DeSimone, 2001; Snyder, 2002).

Using Snyder's hope model, hope has emerged as a potential buffer from negative life events, and therefore has potential to be an incredibly powerful tool to aid our youth. For example, in their validation of the Children's Hope Scale, Curry, Snyder, Cook, Ruby and Rehm (1997) concluded that European-American children high in hope typically have lower levels of depression and higher self-perceptions of athletic ability, physical appearance, social acceptance, and scholastic competence. Furthermore, hope also appears to be implicated in adolescent health outcomes, as children with high hope are more likely to adhere to medical regimens (Berg, Rapoff, Snyder, \& Belmont, 2007), and higher hope levels contribute to less disruptive behavior and higher self-worth (Barnum, Snyder, Rapoff, Mani, \& Thompson, 1998).

While all these positive effects of hope are exciting and promising, the beneficial effect hope has on academics is one that could immediately begin helping Latino youth. Academic troubles are widespread within many Latino populations; as $41 \%$ of Hispanics ages 20 and older in the United States do not have a regular high school diploma (Fry, 2010). In contrast, $23 \%$ of comparably aged blacks and $14 \%$ of whites do not have a regular high school diploma (Fry, 2010). It should be noted, that part of the reason for the high rates of no high school diploma can be attributed to Latinos immigrating into this country without their high school diploma. Thus, part of the reason for the high rates is that they are emigrating from poor countries. Regardless, the area of hope appears to be a potential buffer to these academic problems as hope consistently correlates with greater academic performance in grade school, high school, and college (Snyder, 2002). For example, in college students, Snyder and colleagues (1991) found that students with higher levels of hope tend to set higher goals, perceive that they would be more successful at attaining these goals despite receiving feedback that was not supportive of their estimates, and are more likely to attain higher grades. Furthermore, in a 6-year longitudinal study, Snyder, Shorey, Cheavens, Pulvers, Adams III, and Wiklund (2002) found that differences in the hope levels of students entering college predicted their grade point averages after controlling for entrance examination scores. In addition, higher hope students had a greater likelihood of graduation and a lesser likelihood of having been dismissed from school over the 6-year period relative to low hope students.

The results of these hope theory studies have been promising, especially with regard to the potential for interventions increasing students' hope to improve their academic achievement. However, to date these studies have been conducted with primarily European-American participants. Thus, while it seems logical that hope's effects are universal, not all findings within majority populations transfer well to ethnic/racial minority groups and/or other cultures.

Only recently have researchers have begun to examine hope in Mexican-American youth. A study by Edwards, Ong, \& Lopez, (2007) provides support for the reliability and validity of the Children's Hope Scale in this population, and further, the positive correlation of hope with positive affect, life satisfaction, support from family and friends, and optimism suggests that hope is associated with the same beneficial factors in Latinosas it does European-Americans. As suggested by Stoddard and Garcia (2011), hope could be a protective factor for 
immigrant Latino adolescents; research should examine the ways of integrating it into mental health preventative interventions.

As researchers continue to study the potential benefits of hope in minority populations, another important step is to identify factors that increase hope levels in minorities. One potential factor is a positive ethnic identity-the sense of belonging to an ethnic group (Torres, Yznaga, \& Moore, 2011). Ethnic identity is often viewed as being composed of two parts: exploration and commitment (e.g. Torres et al., 2011). Individuals with high levels of ethnic identity exploration are interested in seeking out and finding out knowledge about their heritage culture. Ethnic identity commitment refers to the sense of attachment or sense of belonging and a personal investment to one's heritage group (Torres et al., 2011). Other researchers (e.g. Umaña-Taylor, Yazedijian, \& Bámaca-Gómez, 2004) have added a third component to ethnic identity, affirmation, which refers to the degree of positive thinking towards one ethnic group.

Research has shown that ethnic identity is associated with various indicators of adjustment, including academic achievement (Altschul, Oyserman, \& Bybee, 2006). Furthermore, Pahl and Way (2006) demonstrated that high levels of ethnic identity tend to counteract the negative effects of discrimination on adjustment among Latinos. It should be noted that while ethnic identity has often been linked with positive adjustment, not all studies have found it to be beneficial (Umaña-Taylor, Gonzales-Backen, \& Guimond, 2009). The distinction between exploration and commitment aspects of ethnic identity could potentially explain these discrepancies. Torres and colleagues (2011) found that when experiencing discrimination, ethnic identity commitment served as a valuable assetfor Latino youth, whereas ethnic identity exploration was associated with increased psychological problems.

In light of the limited number of studies regarding Latino youth hope, the current study had three goals. First, we attempted to determine whether higher hope in Mexican-American students was associated with higher grade point averages (GPA). Given that various studies have shown that increased hope levels is associated with better academic performance in European-American youth samples (Snyder et al., 1991; Snyder, 2002; Snyder et al., 2002), we predicted that that higher hope would be associated with higher GPAs. Secondly, we attempted to shed light on the relationship between ethnic identity and hope. Due to previous research illustrating that ethnic identity is associated with better adjustment and can neutralize the effects of discrimination in Latino samples (Altschul et al., 2006; Pahl \& Way, 2006), we predicted that Latino students given an ethnic pride prime would have higher state hope scores compared to students given a control prime. Lastly, we attempted to determine if there was a difference between ethnic identity commitment and exploration in terms to their relationship to chronic levels of hope. Based on of Torres and colleagues' (2011) findings we predicted that when faced with bicultural stress, ethnic identity commitment would be related to higher levels of chronic hope whereas ethnic pride exploration would be related to lower levels of chronic hope.

\section{Method}

\subsection{Participants}

Twenty-nine Latino youth $(M=16.62, S D=1.14), 9$ males and 20 females, from a high school in South Central Pennsylvania participated in the study. Of the participants, 17 indicated a Mexican-American ethnicity, 10 indicated a Mexican ethnicity, 1 indicated a Puerto Rican ethnicity, and 1 indicated a Dominican-American ethnicity. We recruited participants using flyers posted in the high school and through the school's teacher adviser of Spanish Club. Participants received $\$ 7$ upon successful completion of the study.

\subsection{Procedure}

Once students had received parental consent, we contacted them to set up an appointment to complete the study, which was held in a classroom at their high school. Given the student's schedules, there was anywhere from 1 to 7 participants completing the study at once. At the beginning of each study session, we reviewed the consent form with participants, and allowed them to ask questions. After obtaining consent, we provided each participant with a packet of questionnaires, and sufficient instructions for him/her to complete the questionnaires independently at his/her own pace. Questionnaire forms were securely stapled in uniform order, and assessed demographic information, chronic levels of hope, ethnic identity, and bicultural stress, as described below. Then, the next-to-last form in the packet consisted of a between-group experimental manipulation of Latino pride. Specifically, half the packets (randomly assigned) instructed participant to write a paragraph that was intended to 
elicit feelings of Latino pride, whereas the other half of the packets instructed participants to write a paragraph about their pride in their class year, as a control condition. The last form in the packet was a post-manipulation measure of state hope. These packets were available in English only.

Upon finishing the packet each participant entered a small room attached to the classroom, where we gave him/her a debriefing and an opportunity to ask questions about the current experiment, or experiments in general. We compensated each participant $\$ 7$ for his/her time.

\subsection{Materials}

\subsubsection{Demographic Questionnaire}

In two questionnaires (available in both English and Spanish; one parent, one child) participants and their parents filled out questions answering their social economic status, ethnicity, how long they have resided in the US, their language use at home and at school, student's GPA (optional), and educational/occupational plans for after high school (Table 1).

\subsubsection{Hope Scale}

The Children's Hope Scale (Snyder, 2002) is a six-item measure of children's chronic hope. The scale, based on Snyder's model of hope, contains three items that measure agency (e.g., "I think I am doing pretty well”), and three that measure pathways (e.g., "I can think of many ways to get the things in life that are most important to me"). Participants respond to items on a 6-point scale $(1=$ none of the time, $6=$ all of the time). The alpha coefficient for the Children Hope Scale was .79.

\subsubsection{Latino Identity Scale}

The Latino Identity Scale is an adapted version of the Ethnic Identity Scale (Umaña et al., 2004). The EIS is a 17-item measure of children's ethnic identity. The scale, based on Erikson's and Tajifel's theoretical perspectives, assesses three domains of ethnic identity formation: exploration (e.g., "I have participated in activities that have exposed me to my Latino culture/heritage"), resolution (e.g., "I understand how I feel about being Latino"), and affirmation (e.g., "I am not happy with my Latino culture/heritage"). Our adapted version of the EIS replaced the word "ethnicity" in the EIS to "Latino" to make the measure more personalized to our sample. Participants respond to items on a 4-point scale ( 1 = does not describes me at all, $4=$ describes me very well). The alpha coefficient for the Latino Identity Scale was .75.

\subsubsection{Bicultural Stress Scale}

The Bicultural Stress Scale (Romero \& Roberts, 2003) is a 20-item scale designed to measure the stress within a

Table 1. Correlations between demographic information and pre-manipulation variables.

\begin{tabular}{cccccccccccc}
\hline & Age & Sex & GPA & SES & Exp. & Res. & EI-T & CHS-A & CHS-P & CHS-T & BCS \\
\hline Age & & .09 & .33 & -.03 & .04 & -.05 & .04 & -.19 & -.12 & -.17 & -.02 \\
Sex & .09 & & -.10 & .04 & -.15 & -.13 & -.14 & .01 & .14 & .08 & .07 \\
GPA & .33 & -.10 & & -.10 & .14 & .12 & .13 & .26 & .06 & .19 & .25 \\
SES & -.03 & .04 & -.10 & & .01 & .09 & .02 & -.17 & -.23 & -.21 & .18 \\
Exp. & .04 & -.15 & .13 & .01 & & $.57^{*}$ & $.94^{*}$ & .09 & .17 & .14 & $.51^{*}$ \\
Res. & -.05 & -.13 & .25 & .09 & $.57^{*}$ & & $.80^{*}$ & .31 & $.38^{*}$ & $.37^{*}$ & .12 \\
EI-T & .04 & -.14 & .13 & .02 & $.94^{*}$ & $.80^{*}$ & & .17 & .26 & .23 & $.94^{*}$ \\
CHS-A & -.19 & .01 & .26 & -.17 & .09 & .31 & .17 & & $.70^{*}$ & $.93^{*}$ & -.01 \\
CHS-P & -.12 & .14 & .06 & .23 & .17 & $.38^{*}$ & .26 & $.70^{*}$ & & $.91^{*}$ & -.02 \\
CHS-T & -.17 & .08 & .19 & .21 & .14 & $.37^{*}$ & .23 & $.93^{*}$ & $.91^{*}$ & & -.01 \\
BCS & -.02 & .07 & .25 & .18 & $.51^{*}$ & .12 & $.94^{*}$ & -.01 & -.02 & -.01 & \\
\hline
\end{tabular}

Note. Asterisks indicate a significant correlation, $p<.05$. 
bicultural context in adolescents from Mexican descent. The scale incorporates various questions that address potential stress areas (e.g. social, family, language, etc.), and participants respond to items on a 5-point scale (1 = not at all stressful, 4 = very stressful, $5=$ does not apply). The alpha coefficient for the Bicultural Stress Scale was .85 .

\subsubsection{Ethnic Pride Manipulation}

We randomly assigned participants to one of two conditions. In the ethnic pride condition, a form within the participants' questionnaire packets instructed them to write a paragraph describing what made them proud to be Latino. In the control condition, participants received an identical packet of questionnaires except that this particular form instead instructed participants to write a paragraph describing what made them proud to be a part of their high school class year. To facilitate the task for participants, we included a statement asking them to think about recent times they were proud to be Latino or part of their class year, and what made them proud in those times.

\subsubsection{Post-Manipulation State Hope Scale}

Immediately after the pride manipulation, participants completed the State Hope Scale (Snyder, 2002). Based on Snyder's theory of hope, the 6-item scale measures agency (e.g., "At the present time, I am energetically pursing my goals") and pathway (e.g., "I can think of many ways to reach my current goals") levels of hope at the current moment. Participants respond to items on a 8-point scale $(1=$ definitely false, $8=$ definitely true $)$.

\section{Results}

\subsection{General Information}

We first conducted correlations between the demographic information, ethnic identity, bicultural stress, and chronic hope (Table 1). Next, we analyzed whether demographic information, chronic hope, and state hope were correlated. The analyses revealed that that age, $r=-.45, p=.01$, and chronic hope scores, $r=.41, p=.03$, were significantly correlated to state hope scores. As a result, in all analyses with state hope as a dependent variable, we included age and chronic hope as covariates. Also, during our initial analyses, we found that the affirmation subscale of ethnic identity had nearly no variance, since it was at the maximum possible level for nearly all participants and the remaining few were only one point below the maximum. Hence, although we retained the affirmation items in our computation of total ethnic identity scale, we were unable to examine the affirmation subscale separately in this study.

Separate independent $t$-tests revealed that the control and experimental groups did not significantly differ on sex, age, GPA, SES, bicultural stress, ethnic identity, or chronic levels of hope.

\subsection{GPA and Chronic Hope}

To explore our hypothesis that higher chronic hope is related to higher academic performance, we conducted a correlation analyses between GPA, chronic hope, and chronic hope's subscales. The analyses revealed that the correlation between GPA and total chronic hope, $r=.19, p=.46$, chronic agency, $r=.26, p=.29$, and chronic pathway, $r=.06, p=.81$, were all not significant (Table 1 ). However, only 18 out of the 29 participants provided their GPA. Adding age, sex, socioeconomic status, bicultural stress, and ethnic identity as covariates did not significantly alter the correlation.

\subsection{Ethnic Identity and Chronic Hope}

To test our hypotheses that ethnic identity exploration would be related to lower levels of hope, and that ethnic identity resolution would be related to higher levels of hope, we conducted correlations between exploration, resolution, and chronic hope and ethnic identity (Table 1). The analyses revealed that the resolution subscale of ethnic identity was significantly correlated to chronic hope, $r=.37, p=.05$. Specifically, resolution was significantly correlated to the subcategory of chronic pathway, $r=.38, p=.04$, and marginally correlated to subcategory of chronic agency, $r=.31, p=.11$. Exploration and total ethnic identity scores were not significantly correlated with chronic hope. 


\subsection{The Effects of Boosting Ethnic Pride on State Hope}

To explore our hypothesis that an ethnic boost (i.e., a prime to boost pride in one's Latino ethnicity) would lead to higher state hope relative to a control prime (boosting pride in being a member of one's class year at school), we conducted a one-way analysis of covariance (ANCOVA) with age and chronic hope as covariates, to determine the effect of boost type (ethnic, class) on total state hope scores. The analysis revealed a significant effect of boost type, $F(1,25)=6.21, p=.02, \eta_{p}^{2}=.20$, as the ethnic boost group $(M=40.07, S E=.59)$ had significantly higher total state scores than the control group $(M=37.93, S E=.61)$.

Given the significant main effect of boost type on state hope scores, we next explored how boost affected the subscales of state hope by conducting separate one-way ANCOVAS to determine how boost type (ethnic, class) affects agency and pathway state hope scores (Figure 1). The analyses revealed a significant main effect of boost type on state agency score, $F(1,25)=14.71, p=.001, \eta_{p}^{2}=.37$, as the ethnic boost group $(M=20.05$, $S E=.34)$ had significantly higher agency scores than control group $(M=18.17, S E=.35)$. Furthermore, the analysis revealed that the main effect of boost type was not significant on state pathway scores, $F(1,25)=0.16$, $p=.69, \eta_{p}^{2}=.01$. Figure 1 displays the corrected means from these analyses.

The significant correlation between ethnic identity and chronic hope suggested that differences in level of chronic ethnic identity might possibly moderate the effect of the ethnic boost on state hope. That is, perhaps the effect of the manipulation on state hope depends on the positivity/negativity of each individual's ethnic identity being brought to mind by the ethnic boost prime. The ANCOVAs described above were repeated, but this time also including ethnic identity scores and their interaction with boost type in the model. The effect of the interaction between ethnic identity and boost type on total state hope scores was not significant, $F(1,23)=0.38, p$ $=.54$. Likewise, the interaction between ethnic identity and boost type showed no significant effects on the separate subscales of state hope.

\section{Discussion}

The present study found that participants in the ethnic boost group had higher state hope scores than those in the class boost group, supporting our hypothesis. Furthermore, we found that this difference in state hope scores was due to the ethnic boost increasing stage hope agency scores more than the control condition. In terms of academic achievement, we found that chronic hope levels were not associated with higher GPA, although there are some limiting factors to our study that could explain this null result. Lastly, our hypothesis that ethnic identity resolution would be related to higher levels chronic hope was supported. However, our hypothesis that ethnic identity exploration would be related to lower chronic hope levels was not supported.

Our main finding that ethnic boost group participants had higher state hope scores fits in with those studies that have found beneficial effects of ethnic identity (e.g. Altschul et al., 2006; Pahl \& Way, 2006). Thus, our

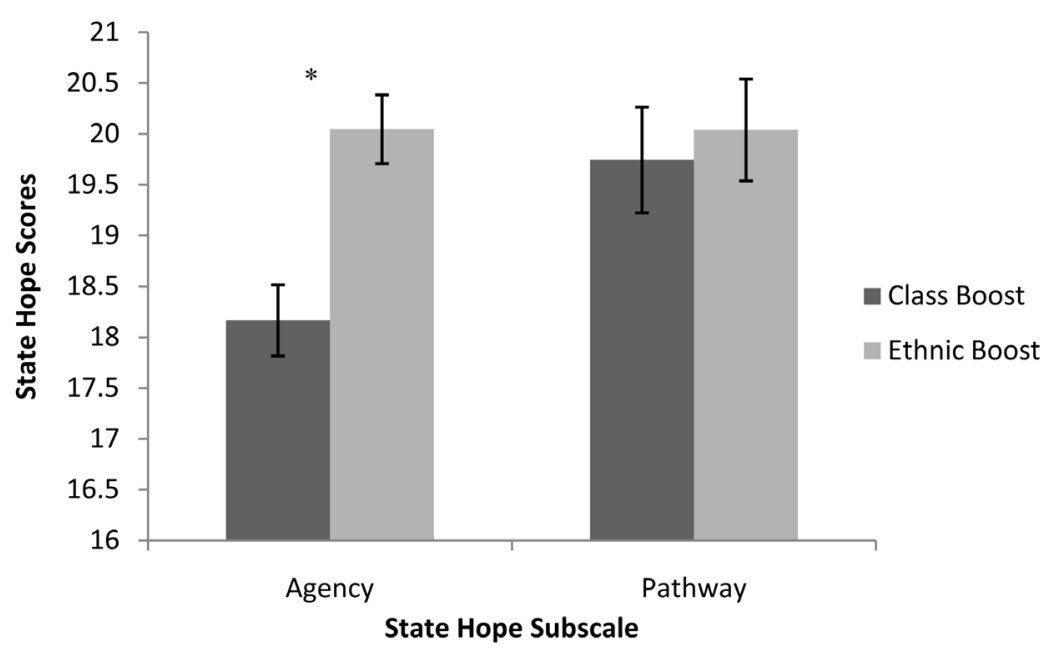

Figure 1. Corrected mean (+/- $1 S E)$ state hope subscale scores as a function of boost type, controlling for age and the score for the chronic level of the same hope subscale. 
study suggests that ethnic identity is a factor that contributes to high hope in Latino youth. In line with this explanation, we found that the resolution subscale of ethnic identity was positively correlated with chronic hope. That is, Latino youth who had resolved their views on their ethnicity had higher chronic hope scores. Whether ethnic identity and hope is a one-way relationship needs to be determined, as it is also possible that hope contributes to a more positive ethnic identity. Future studies could explore this possibility by using a hope manipulation to determine whether hope increases Latino youths' ethnic identity. Regardless, our study demonstrated the powerful effect ethnic identity in bolstering hope.

Interestingly, we found that the ethnic boost increased the hope subscale of agency, but not pathway. That is, the ethnic boost did not affect participants' confidence in their ability to create routes to achieve their goals (pathway), but did increase their confidence in their ability to use these pathways to achieve their goals (agency). Thus, the ethnic boost affected the motivation aspect of hope. Future studies need to explore why the ethnic boost affects only agency hope, and not pathway hope.

Given hope's beneficial effects in nearly all domains (see Snyder, 2002 for review), our findings are extremely promising and emphasize how important it is that schools, teachers, and parents accentuate Latino youth's pride in their culture. The lack of interaction between ethnic identity and boost type suggests that regardless of chronic levels ethnic identity, an ethnic boost increases state hope. Thus, our findings illustrate the influential nature of accentuating one's ethnic pride. With ethnic pride being so impactful, future programs designed to increase hope in Latino youth should consider incorporating an ethnic pride component. At the same time, the lack of interaction between ethnic identity and boost type may result from a limitation specific to our study. To recruit participants, we utilized the school's Spanish teacher and Spanish Club teacher advisor. As a result, most of the students that participated were a part of the Spanish Club, and thus, most likely proud of their identity. Thus, it is possible that we were unable to establish an interaction because ethnic identity levels were high and had limited variability begin with. If so, this also presents the possibility that the generality of our sample is limited to Latino students who have a particularly strong, positive identification with their ethnic group. Future experiments with larger variation in ethnic identity could help determine if an ethnic boost increases state hope in Latino youth with lower ethnic identity.

While we were able to show a link between ethnic identity and hope, we were unable to demonstrate a positive relationship between GPA and chronic levels of hope. Our finding does not fall within the literature demonstrating the positive effect of hope and academic performance (Snyder et al., 2002). A likely explanation for our null results is that our sample was too small to detect the expected relationship. Due to our desire to help students feel comfortable participating in the project and be respectful of their privacy, we made our request for GPA optional, and only 18 of 29 participants opted to report it. Furthermore, because the request for GPA was optional, it is possible that only those with higher GPAs reported their GPAs. In line with this explanation, the average GPA was $89.52 \%$. Another less likely explanation is that the link between hope and academic performance does not exist in Latino youth populations as it does in majority populations. Future studies with Latino youth populations are crucial in identifying whether link between GPA and Latino youth is similar to that of majority populations.

The last goal of the current experiment was to determine the differential associations of aspects of ethnic identity with chronic hope levels. In line with the Torres and colleagues (2011), we found that ethnic identity commitment/resolution, was significantly positively correlated with hope. As noted previously, this adds support to our main finding, that a positive ethnic identity, (whether reflected in chronic levels or due to a prime that temporarily boosts pride in ethnic identity) is associated with higher levels of hope.

We had also predicted that the exploration aspect of ethnic identity would be negatively related to hope levels. Although exploration significantly correlated with reports of experiencing more bicultural stress, exploration was not related to hope (and neither was bicultural stress). Our null finding is inconsistent with the literature showing negative effects of ethnic identity exploration (e.g. Torres et al., 2011). It is possible that the limited sample size and variability within our sample resulted in an inability to detect the expected negative association. Yet it is also possible that ethnic exploration might be differentially associated with specific negative, positive, or neutral aspects of adjustment, and that its associations may dependent on other features of the sample or individual. Thus, the possibility that ethnic exploration may not be universally linked with features that are detrimental to adjustment remains an important area to explore in future studies that should include, in addition to measures of hope, a wider range of psychological adjustment variables. These studies should also include a large enough and sufficiently diverse sample to examine under what circumstances and for what particular aspects of 
psychological adjustment the implications of ethnic exploration might be detrimental, neutral, or beneficial.

Future studies will be required to clarify the robustness of our findings. While our ability to obtain several significant predicted effects in such a small sample is consistent with detection of robust relationships, other effects that we predicted were not found (e.g. high ethnic identity) in our sample. Future studies of these phenomena will require larger sample sizes and greater variability in ethnic identity to overcome the limitations of our study in terms of power and generalizability.

\section{Conclusion}

In sum, our study was able to identity ethnic identity as a factor that contributes to both state and chronic levels of hope. Given this finding, it is extremely important that we foster Latino youth's ethnic identity, and encourage them to celebrate and embrace their ethnicity. Furthermore, future programs looking to increase hope in Latinos youth should consider incorporating an ethnic identity portion. In terms of academic performance, we were unable to establish a link between chronic hope levels and GPA; however this could be related to limitations in our design. Ultimately, future research needs to continue investigating factors that contribute to hope in minority populations, and whether the hope benefits in majority populations are also present in minority populations.

\section{References}

Altschul, I., Oyserman, D., \& Bybee, D. (2006). Racial-Ethnic Identity in Mid-Adolescence: Content and Change as Predictors of Academic Achievement. Child Development, 77, 1155-1169. http://dx.doi.org/10.1111/j.1467-8624.2006.00926.x

Barnum, D. D., Snyder, C. R., Rapoff, M. A., Mani, M. M., \& Thompson, R. (1998). Hope and Social Support in the Psychological Adjustment of Children Who Have Survived Burn Injuries and Their Matched Controls. Children's Health Care, 27, 15-30. http://dx.doi.org/10.1207/s15326888chc2701_2

Berg, C. J., Rapoff, M. A., Snyder, C. R., \& Belmont, J. M. (2007). The Relationship of Children’s Hope to Pediatric Asthma Treatment Adherence. The Journal of Positive Psychology, 2, 176-184. http://dx.doi.org/10.1080/17439760701409629

Chang, E. C., \& DeSimone, S. L. (2001). The Influence of Hope on Appraisals, Coping, and Dysphoria: A Test of Hope Theory. Journal of Social and Clinical Psychology, 20, 117-129. http://dx.doi.org/10.1521/jscp.20.2.117.22262

Curry, L. A., Snyder, C. R., Cook, D. L., Ruby, B. C., \& Rehm, M. (1997). Role of Hope in Academic and Sport Achievement. Journal of Personality and Social Psychology, 73, 1257-1267. http://dx.doi.org/10.1037/0022-3514.73.6.1257

Edwards, L. M., Ong, A. D., \& Lopez, S. J. (2007). Hope Measurement in Mexican American Youth. Hispanic Journal of Behavioral Sciences, 29, 225-241. http://dx.doi.org/10.1177/0739986307299692

Fry, R. (2010). Hispanics, High School Dropouts and the GED. Washington, DC: Pew Hispanic Center.

Lopez, M. H., Morin, R., \& Taylor, P. (2009). Illegal Immigration Backlash Worries, Divides Latinos. Washington, DC: Pew Research Center.

McClure, E. B., \& Pine, D. S. (2006). Social Anxiety and Emotion Regulation: A Model for Developmental Psychopathology Perspectives on Anxiety Disorders. In D. Cicchetti, D. J. Cohen, D. Cicchetti, \& D. J. Cohen (Eds.), Developmental Psychopathology, Vol 3: Risk, Disorder, and Adaptation (2nd ed., pp. 470-502), Hoboken, NJ: John Wiley \& Sons Inc.

Pahl, K., \& Way, N. (2006). Longitudinal Trajectories of Ethnic Identity among Urban Black and Latino Adolescents. Child Development, 77, 1403-1415. http://dx.doi.org/10.1111/j.1467-8624.2006.00943.x

Romero, A. J., \& Roberts, R. E. (2003). Stress within a Bicultural Context for Adolescents of Mexican Descent. Cultural Diversity and Ethnic Minority Psychology, 9, 171-184. http://dx.doi.org/10.1037/1099-9809.9.2.171

Snyder, C. R. (1994). The Psychology of Hope: You Can Get There from Here. New York, NY: Free Press.

Snyder, C. R. (2002). Hope Theory: Rainbows in the Mind. Psychological Inquiry, 13, 249-275. http://dx.doi.org/10.1207/S15327965PLI1304 01

Snyder, C. R., Harris, C., Anderson, J. R., Holleran, S. A., Irving, L. M., Sigmon, S. T., \& Harney, P. (1991). The Will and the Ways: Development and Validation of an Individual-Differences Measure of Hope. Journal of Personality and Social Psychology, 60, 570-585. http://dx.doi.org/10.1037/0022-3514.60.4.570

Snyder, C. R., Shorey, H. S., Cheavens, J., Pulvers, K., Adams, V., \& Wiklund, C. (2002). Hope and Academic Success in College. Journal of Educational Psychology, 94, 820-826. http://dx.doi.org/10.1037/0022-0663.94.4.820

Stoddard, S. A., \& Garcia, C. M. (2011). Hopefulness among Non-US-Born Latino Youth and Young Adults. Journal of Child and Adolescent Psychiatric Nursing, 24, 216-222. http://dx.doi.org/10.1111/j.1744-6171.2011.00307.x

Torres, L., Yznaga, S. D., \& Moore, K. M. (2011). Discrimination and Latino Psychological Distress: The Moderating Role of Ethnic Identity Exploration and Commitment. American Journal of Orthopsychiatry, 81, 526-534. 
D. Yager-Elorriaga et al.

http://dx.doi.org/10.1111/j.1939-0025.2011.01117.x

Umaña-Taylor, A. J., Gonzales-Backen, M. A., \& Guimond, A. B. (2009). Latino Adolescents' Ethnic Identity: Is There a Developmental Progression and Does Growth in Ethnic Identity Predict Growth in Self-Esteem? Child Development, 80, 391-405. http://dx.doi.org/10.1111/j.1467-8624.2009.01267.x

Umaña-Taylor, A. J., Yazedjian, A., \& Bámaca-Gómez, M. (2004). Developing the Ethnic Identity Scale Using Eriksonian and Social Identity Perspectives. Identity: An International Journal of Theory and Research, 4, 9-38.

http://dx.doi.org/10.1207/S1532706XID0401_2 
Scientific Research Publishing (SCIRP) is one of the largest Open Access journal publishers. It is currently publishing more than 200 open access, online, peer-reviewed journals covering a wide range of academic disciplines. SCIRP serves the worldwide academic communities and contributes to the progress and application of science with its publication.

Other selected journals from SCIRP are listed as below. Submit your manuscript to us via either submit@scirp.org or Online Submission Portal.
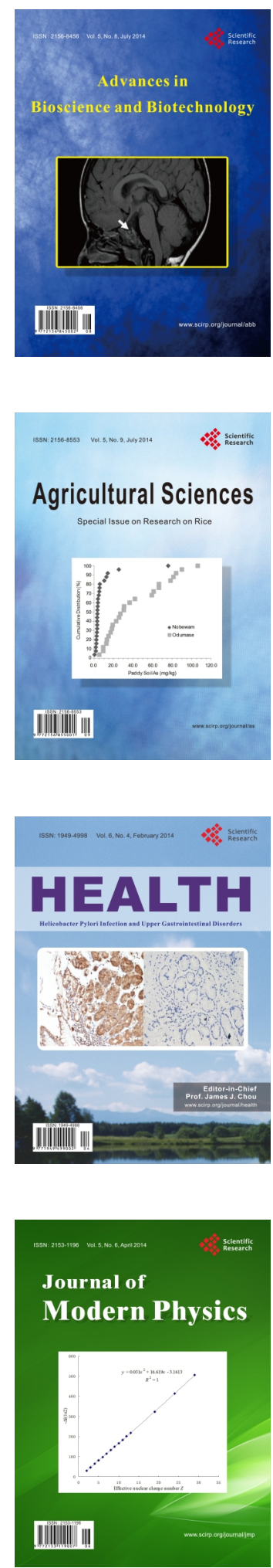
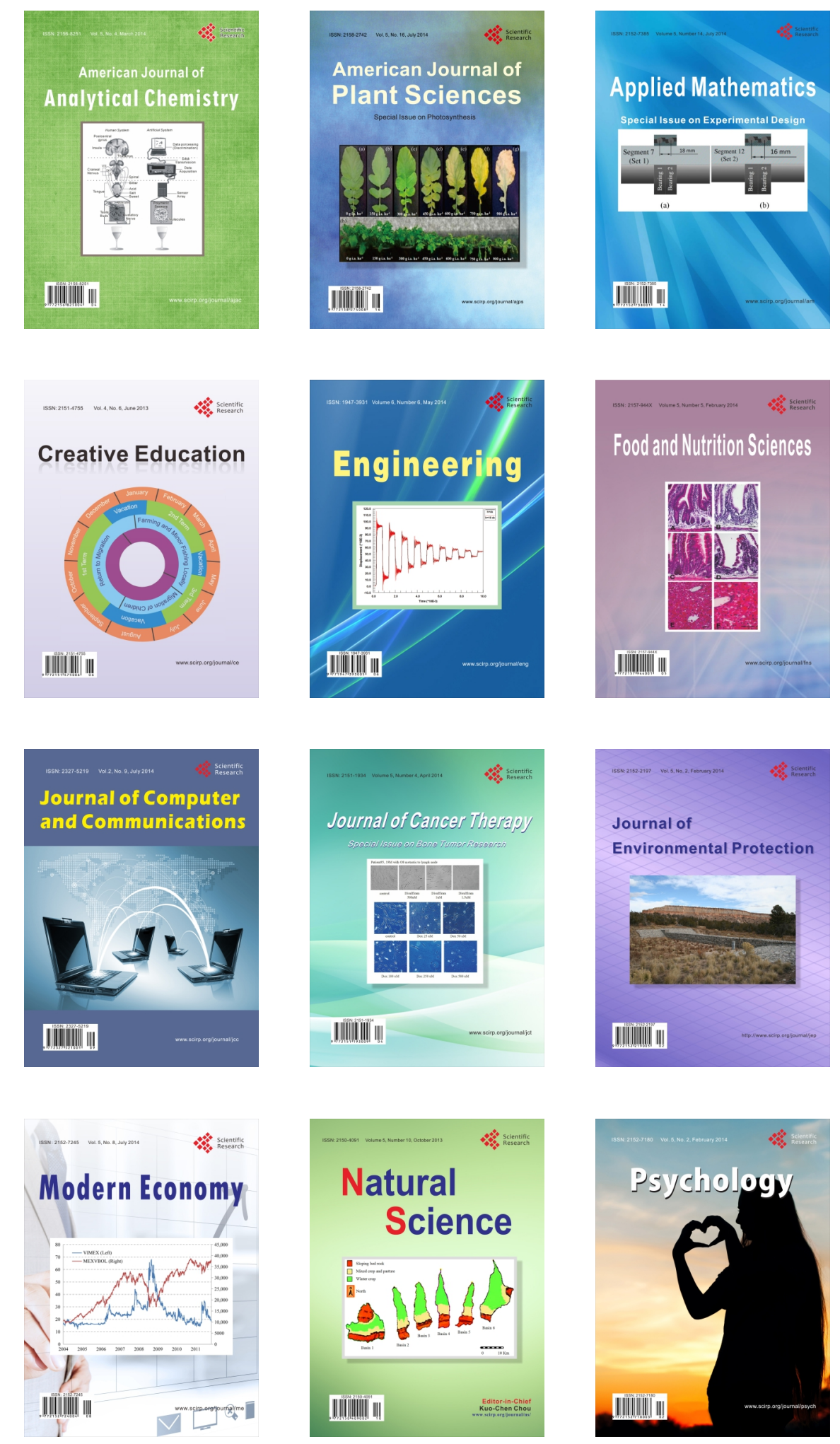